3. N. J. Divinsky, Rings and radicals, Univ. of Toronto Press, Toronto, 1965.

4. T. Anderson, N. Divinsky and A. Sulinski, Hereditary radicals in associative and alternative rings, Canad. J. Math. 17 (1965), 594-603.

5. A. E. Hoffman, The constructions of the general theory of radicals, Ph.D. Thesis, University of Nebraska, Lincoln, 1966.

UNIVERSITY OF NEBRASKa AND

University of Southern California

\title{
ON COHOMOLOGICAL TRIVIALITY
}

\section{H. ONISHI}

Let $G$ be a finite group and $A$ be a $G$-module. It is well known that if for some two consecutive dimensions the cohomology groups $H^{r}(H, A)$ are trivial for all subgroups $H$ of $G$, then $H^{r}(H, A)$ are trivial for all dimensions $r$ and for all subgroups $H$ of $G$. This note is to point out the following generalization.

Theorem. Let $G$ be a finite group and $A$ be a G-module. If for some integer $k$ and for some odd positive integer $d, H^{k}(H, A)$ and $H^{k+d}(H, A)$ are trivial for all subgroups $H$ of $G$, then $H^{r}(H, A)$ are trivial for all integers $r$ and for all subgroups $H$ of $G$.

Proof. As usual we proceed by induction on the order $n=|G|$. The theorem is trivial for $n=1$. Suppose $n>1$ and assume the truth of the theorem for all groups of order $<n$. In particular, we may assume that $H^{r}(H, A)=0$ for every dimension $r$ and for every proper subgroup $H$ of $G$. If $n$ is not a prime power, then every Sylow subgroup of $G$ is a proper subgroup and the conclusion follows by a wellknown result.

Suppose now $n$ is a prime power, so that $G$ is solvable. Let $H$ be a proper normal subgroup of $G$ such that the quotient group $G / H$ is cyclic. Since $H^{r}(H, A)=0$ for every $r$, we have the Fundamental Exact Sequence of Hochschild and Serre [2];

$$
0 \rightarrow H^{r}\left(G / H, A^{H}\right) \rightarrow H^{r}(G, A) \rightarrow H^{r}(H, A)
$$

for every $r>0$. Since the last term is trivial, this says that $H^{r}\left(G / H, A^{\boldsymbol{H}}\right)$ $\cong H^{r}(G, A)$ for every $r>0$. By dimension shifting we could have assumed that $k>0$. Thus we have that $H^{k}\left(G / A, A^{H}\right)$ $=H^{k+d}\left(G / H, A^{H}\right)=0$. Since $G / H$ is cyclic and $d$ is odd, we obtain

Received by the editors September 1, 1966. 
that $H^{r}\left(G / H, A^{H}\right)=0$ for every $r$. But then by the isomorphism $H^{r}(G, A)=0$ for every $r>0$. Once we have this, then we obtain that $H^{r}(G, A)=0$ for every $r \leqq 0$ as well. Q.E.D.

The theorem suggests the following conjecture.

Conjecture. Let $G$ be a finite group. Let $A$ and $B$ be $G$-modules and let $f: A \rightarrow B$ be a $G$-homomorphism. If $f$ induces isomorphisms

$$
H^{r}(H, A) \cong H^{r}(H, B)
$$

for some two dimensions that differ by an odd integer and for all subgroups $H$ of $G$, then $f$ induces isomorphisms for all dimensions $r$ and for all subgroups $H$ of $G$.

The theorem proved is the special case of the conjecture when $B$ is trivial. L. Evens [1] proved the conjecture when the difference of the dimensions is 1 . The conjecture is easily seen to be true when $G$ has a cohomological period by reducing it to the case when $G$ is a p-group.

\section{REFERENCES}

1. L. Evens, An extension of Tate's theorem on cohomological triviality, Proc. Amer. Math. Soc. 16 (1965), 289-291.

2. G. Hochschild and J.-P. Serre, Cohomology of group extensions, Trans. Amer. Math. Soc. 74 (1953), 110-134.

City College of New York 